See discussions, stats, and author profiles for this publication at: https://www.researchgate.net/publication/277608346

\title{
Spectral Properties of Fourth Order Differential Operators with Periodic and
} Antiperiodic Boundary Conditions

Article in Results in Mathematics · March 2015

DOI: 10.1007/500025-015-0454-2

\section{CITATIONS}

2

3 authors, including:

Hikmet Gunes

Inonu University

1 PUBLICATION 2 CITATIONS

SEE PROFILE
161

Nazim B. Kerimov

Mersin University,Mersin, Turkey

48 PUBLICATIONS 516 CITATIONS

SEE PROFILE

Some of the authors of this publication are also working on these related projects: 


\title{
Spectral Properties of Fourth Order Differential Operators with Periodic and Antiperiodic Boundary Conditions
}

\author{
Hikmet Gunes, Nazim B. Kerimov and Ufuk Kaya
}

Abstract. In this paper, we consider the following periodic and antiperiodic problem

$$
\begin{gathered}
y^{\text {iv }}+p_{2}(x) y^{\prime \prime}+p_{1}(x) y^{\prime}+p_{0}(x) y=\lambda y, \quad 0<x<1, \\
y^{(s)}(1)-(-1)^{\sigma} y^{(s)}(0)=0, \quad s=\overline{0,3},
\end{gathered}
$$

where $\lambda$ is a spectral parameter; $p_{j}(x) \in L_{1}(0,1), j=0,1, p_{2}(x) \in$ $W_{1}^{1}(0,1)$ with $\int_{0}^{1} p_{2}(\xi) d \xi=0$ are complex-valued functions and $\sigma=0,1$. The boundary conditions of this problem are periodic-antiperiodic boundary conditions and it is well known that they are regular but not strongly regular. Asymptotic formulae for eigenvalues and eigenfunctions of the considered boundary value problem are established. Under the condition

$$
\left(p_{2}(1)-p_{2}(0)-2 c_{1}\right)\left(p_{2}(1)-p_{2}(0)+2 c_{1}\right) \neq 0,
$$

it is proved that all the eigenvalues (except for finite number) are simple, where $c_{1}=\int_{0}^{1} p_{1}(\xi) d \xi$. Furthermore, we prove that the system of root functions of this spectral problem forms a basis in the space $L_{p}(0,1)$, $1<p<\infty$, when $p_{1}(1)=p_{1}(0) ; p_{2}^{(s)}(1)=p_{2}^{(s)}(0), s=0,1 ; p_{j}(x) \in$ $W_{1}^{j}(0,1), j=0,1,2 ; c_{1} \neq 0$. Also, it is shown that this basis is unconditional for $p=2$.

Mathematics Subject Classification. 34B05, 34L20, 34L10.

Keywords. Fourth order eigenvalue problem, periodic and antiperiodic boundary conditions, asymptotic behavior of eigenvalues and eigenfunctions, basis properties of the system of root functions. 


\section{Introduction}

Henceforward, by $L$ we denote a differential operator generated by the differential expression

$$
l(y)=y^{\mathrm{iv}}+p_{2}(x) y^{\prime \prime}+p_{1}(x) y^{\prime}+p_{0}(x) y, \quad x \in(0,1),
$$

where $p_{j}(x) \in L_{1}(0,1), j=0,1, p_{2}(x) \in W_{1}^{1}(0,1)$ with $\int_{0}^{1} p_{2}(\xi) d \xi=0$, are complex-valued functions and the periodic-antiperiodic boundary conditions

$$
U_{s}(y) \equiv y^{(s)}(1)-(-1)^{\sigma} y^{(s)}(0)=0, \quad s=\overline{0,3}, \quad \sigma=0,1 .
$$

It is easy to verify that boundary conditions (2) are regular, but not strongly regular.

In this paper, the structures of eigenvalues and root functions of the operator $L$ are given under the condition

$$
\left(p_{2}(1)-p_{2}(0)-2 c_{1}\right)\left(p_{2}(1)-p_{2}(0)+2 c_{1}\right) \neq 0 .
$$

Asymptotic formulae for eigenvalues and root functions are obtained. Moreover, the basicity of the root functions system is established in the space $L_{p}(0,1), 1<p<\infty$ when $p_{1}(1)=p_{1}(0) ; p_{2}^{(s)}(1)=p_{2}^{(s)}(0), s=0,1 ; p_{j}(x) \in$ $W_{1}^{j}(0,1), j=0,1,2 ; c_{1} \neq 0$, where

$$
c_{1}=\int_{0}^{1} p_{1}(\xi) d \xi .
$$

In [13-15], Kerimov and Kaya have investigated the following problem

$$
\begin{gathered}
y^{\mathrm{iv}}+p_{2}(x) y^{\prime \prime}+p_{1}(x) y^{\prime}+p_{0}(x) y=\lambda y, \quad 0<x<1, \\
y^{\prime \prime \prime}(1)-(-1)^{\sigma} y^{\prime \prime \prime}(0)+\alpha_{3,2} y^{\prime \prime}(0) \alpha_{3,1} y^{\prime}(0)+\alpha_{3,0} y(0)=0, \\
y^{\prime \prime}(1)-(-1)^{\sigma} y^{\prime \prime}(0)+\alpha_{2,1} y^{\prime}(0) \alpha_{2,0} y(0)=0, \\
y^{\prime}(1)-(-1)^{\sigma} y^{\prime}(0)+\alpha_{1,0} y(0)=0, \\
y(1)-(-1)^{\sigma} y(0)=0
\end{gathered}
$$

in various conditions. However, there is no case reduced to the periodicantiperiodic boundary conditions. Indeed, the basicity properties of a fourth order periodic-antiperiodic problem are firstly investigated in this paper.

In $[8,17,29]$, it has been studied basicity of the system of root functions of differential operators with strongly regular boundary conditions. Besides, basicity of the system of root functions of differential operators with regular (but not strongly) boundary conditions is studied in $[3-7,9,11,16,18-28,31-$ 35]. For the Sturm-Liouville operator with periodic (antiperiodic) boundary conditions, first examples of potentials providing divergence of corresponding spectral expansions were constructed in [21] and [7]. More information about these studies was given in [13-15].

Let us define the number $\gamma_{0}$ as follows. Assume that $\gamma_{0}$ is any fixed number satisfying the equality

$$
\gamma_{0}^{2}=(-1)^{\sigma}\left(p_{2}(1)-p_{2}(0)-2 c_{1}\right)\left(p_{2}(1)-p_{2}(0)+2 c_{1}\right) .
$$


The following assertions are the basic results of this paper:

Theorem 1. Let $p_{0}(x), p_{1}(x) \in L_{1}(0,1), p_{2}(x) \in W_{1}^{1}(0,1)$ be arbitrary complexvalued functions and $\gamma_{0} \neq 0$. Then all eigenvalues of differential operator (1)(2) except for a finite number, are simple and form two infinite sequences $\lambda_{n, 1}$ and $\lambda_{n, 2}, n=1,2, \ldots$ Moreover, for sufficiently large numbers $n$, the asymptotic formula

$$
\lambda_{n+n_{j}, j}=((2 n-\sigma) \pi)^{4}\left\{1+\frac{(-1)^{j} \gamma_{0}}{2((2 n-\sigma) \pi)^{3}}+O\left(n^{-3} \varepsilon_{n}\right)\right\}
$$

is valid, where $j=1,2$ and $n_{1}, n_{2}$ are certain integers. Furthermore, for sufficiently large numbers $n$, the corresponding eigenfunctions $u_{n, 1}(x)$ and $u_{n, 2}(x)$, $n=1,2, \ldots$, have the following asymptotic formulae:

$$
\begin{aligned}
u_{n+n_{j}, j}(x)= & \left(2 c_{1}+\left(p_{2}(1)-p_{2}(0)\right)\right) \cos (2 n-\sigma) \pi x \\
& +(-1)^{j} \gamma_{0} \sin (2 n-\sigma) \pi x+O\left(\varepsilon_{n}\right)
\end{aligned}
$$

where

$$
\begin{aligned}
\varepsilon_{n}= & \left|\int_{0}^{1}\left(2 p_{1}(\xi)-p_{2}^{\prime}(\xi)\right) e^{2(2 n-\sigma) \pi i \xi} d \xi\right| \\
& +\left|\int_{0}^{1}\left(2 p_{1}(\xi)-p_{2}^{\prime}(\xi)\right) e^{-2(2 n-\sigma) \pi i \xi} d \xi\right|+n^{-1}
\end{aligned}
$$

Theorem 2. Let $p_{j}(x) \in W_{1}^{j}(0,1), j=0,1,2, p_{2}^{(s)}(1)=p_{2}^{(s)}(0), s=0,1$ and $p_{1}(1)=p_{1}(0)$. Then the system of root functions of differential operator (1)-(2) forms a basis in the space $L_{p}(0,1), 1<p<\infty$, and this basis is unconditional for $p=2$. In addition, the asymptotic formulae for the eigenfunctions can be written by the following form:

$$
u_{n+n_{j}, j}(x)=\cos (2 n-\sigma) \pi x+(-1)^{j} i^{1-\sigma} \sin (2 n-\sigma) \pi x+O\left(n^{-1}\right)
$$

in this case.

Corollary 1. Assume that all conditions of Theorem 2 be fulfilled. Then $n_{1}+$ $n_{2}=1-\sigma$ and we can choose $n_{1}=0$ and $n_{2}=1-\sigma$.

\section{Some Auxiliary Results}

Let

$$
S_{0}=\left\{\rho \in \mathbb{C}: 0 \leq \arg \rho \leq \frac{\pi}{4}\right\}
$$

where $\mathbb{C}$ is the set of complex numbers. We denote by $\omega_{k}, k=\overline{1,4}$, different 4 -th roots of -1 . It is known that the numbers $\omega_{k}, k=\overline{1,4}$, can be ordered in such a way that for all $\rho \in S_{0}$ the inequalities 


$$
\Re\left(\rho \omega_{1}\right) \leq \Re\left(\rho \omega_{2}\right) \leq \Re\left(\rho \omega_{3}\right) \leq \Re\left(\rho \omega_{4}\right)
$$

hold, where $\Re(z)$ means the real parts of $z$ (see [30, Chapter II, §4.2]). Henceforward, the numbers $\omega_{k}, k=\overline{1,4}$, will be such that for all $\rho \in S_{0}$ the inequalities (10) are valid. In this case, it is proved that the numbers $\omega_{k}, k=\overline{1,4}$, can be determined using the equalities (see [30, Chapter II, §4.8])

$$
\omega_{1}=e^{3 \pi i / 4}, \quad \omega_{2}=e^{-3 \pi i / 4}, \quad \omega_{3}=e^{\pi i / 4}, \quad \omega_{4}=e^{-\pi i / 4} .
$$

It is easy to see that

$$
\omega_{1}=-\omega_{4}, \quad \omega_{2}=-\omega_{3} .
$$

Lemma 1. [15] In $S_{0}$, the following inequalities are valid:

$$
\Re\left(\rho \omega_{1}\right) \leq-\frac{\sqrt{2}}{2}|\rho|, \quad \Re\left(\rho \omega_{4}\right) \geq \frac{\sqrt{2}}{2}|\rho| .
$$

Consider the domain obtained from the sector $S_{0}$ [see (9)] by a translation $\rho \rightarrow \rho-c$, where $c$ is a fixed complex number. This new sector with its vertex at the point $\rho=-c$ will be denoted by $T_{0}$. For the new sector $T_{0}$ the inequalities (10) and (13) will be rewritten in the forms

$$
\begin{aligned}
& \Re\left((\rho+c) \omega_{1}\right) \leq \Re\left((\rho+c) \omega_{2}\right) \leq \Re\left((\rho+c) \omega_{3}\right) \leq \Re\left((\rho+c) \omega_{4}\right), \\
& \Re\left((\rho+c) \omega_{1}\right) \leq-\frac{\sqrt{2}}{2}|\rho+c|, \quad \Re\left((\rho+c) \omega_{4}\right) \geq \frac{\sqrt{2}}{2}|\rho+c| .
\end{aligned}
$$

Fix such a domain $T_{0}$. For $\rho \in T_{0}$, the equation

$$
l(y)+\rho^{4} y=0
$$

has four linearly independent solutions $y_{k}(x, \rho), k=\overline{1,4}$ (see [30, Chapter II, $\S 4.5-4.6])$. These solutions are regular if $|\rho|$ is large enough. The derivatives satisfy the integro-differential equations

$$
\begin{aligned}
\frac{d^{s} y_{k}(x, \rho)}{d x^{s}}= & \rho^{s} \omega_{k}^{s} e^{\rho \omega_{k} x}+\frac{1}{4 \rho^{3}} \int_{0}^{x} \frac{\partial^{s} K_{1}(x, \xi, \rho)}{\partial x^{s}} q(\xi) y_{k}(\xi) d \xi \\
& -\frac{1}{4 \rho^{3}} \int_{x}^{1} \frac{\partial^{s} K_{2}(x, \xi, \rho)}{\partial x^{s}} q(\xi) y_{k}(\xi) d \xi, \quad s=\overline{0,3},
\end{aligned}
$$

where

$$
K_{1}(x, \xi, \rho)=\sum_{\alpha=1}^{k} \omega_{\alpha} e^{\rho \omega_{\alpha}(x-\xi)}, \quad K_{2}(x, \xi, \rho)=\sum_{\alpha=k+1}^{4} \omega_{\alpha} e^{\rho \omega_{\alpha}(x-\xi)} .
$$

Moreover, it is known (see [30, Chapter II, §4.5]) that

$$
\frac{d^{s} y_{k}(x, \rho)}{d x^{s}}=\rho^{s} e^{\rho \omega_{k} x} z_{k, s}(x, \rho),
$$

where $z_{k, s}(x, \rho)$ is analytic function of $\rho$ and it satisfies

$$
z_{k, s}(x, \rho)=\omega_{k}^{s}+O\left(\rho^{-1}\right), \quad k=\overline{1,4}, \quad s=\overline{0,3} .
$$


From (17)-(19), we have

$$
\begin{aligned}
z_{k, s}(x, \rho)= & \omega_{k}^{s}+\frac{\omega_{k}^{s+1}}{4 \rho} \int_{0}^{x} \sum_{j=0}^{2} \frac{p_{j}(\xi)}{\rho^{2-j}} z_{k, j}(\xi, \rho) d \xi \\
& +\frac{1}{4 \rho} \sum_{\alpha=1}^{k-1} \omega_{\alpha}^{s+1} \int_{0}^{x} e^{\rho\left(\omega_{\alpha}-\omega_{k}\right)(x-\xi)} \sum_{j=0}^{2} \frac{p_{j}(\xi)}{\rho^{2-j}} z_{k, j}(\xi, \rho) d \xi \\
& -\frac{1}{4 \rho} \sum_{\alpha=k+1}^{4} \omega_{\alpha}^{s+1} \int_{x}^{1} e^{\rho\left(\omega_{\alpha}-\omega_{k}\right)(x-\xi)} \sum_{j=0}^{2} \frac{p_{j}(\xi)}{\rho^{2-j}} z_{k, j}(\xi, \rho) d \xi
\end{aligned}
$$

Note that, by (14), we have

$$
\Re\left(\rho\left(\omega_{\alpha}-\omega_{\beta}\right)\right)=\Re\left((\rho+c)\left(\omega_{\alpha}-\omega_{\beta}\right)\right)-\Re\left(c\left(\omega_{\alpha}-\omega_{\beta}\right)\right) \leq 2|c|,
$$

where $1 \leq \alpha \leq \beta \leq 4$. From here and (20) we obtain

$$
\begin{aligned}
& \int_{0}^{x} p_{j}(\xi) z_{k, j}(\xi, \rho) e^{\rho\left(\omega_{\alpha}-\omega_{k}\right)(x-\xi)} d \xi=O(1), \quad \alpha \leq k ; \\
& \quad \int_{x}^{1} p_{j}(\xi) z_{k, j}(\xi, \rho) e^{\rho\left(\omega_{\alpha}-\omega_{k}\right)(x-\xi)} d \xi=O(1), \quad \alpha \geq k,
\end{aligned}
$$

where $k=\overline{1,4}$ and $j=0,1,2$. Consequently, it follows from (21) that

$$
\begin{aligned}
z_{k, s}(x, \rho)= & \omega_{k}^{s}+\frac{\omega_{k}^{s+1}}{4 \rho} \int_{0}^{x} \sum_{j=1}^{2} \frac{p_{j}(\xi)}{\rho^{2-j}} z_{k, j}(\xi, \rho) d \xi \\
& +\frac{1}{4 \rho} \sum_{\alpha=1}^{k-1} \omega_{\alpha}^{s+1} \int_{0}^{x} e^{\rho\left(\omega_{\alpha}-\omega_{k}\right)(x-\xi)} \sum_{j=1}^{2} \frac{p_{j}(\xi)}{\rho^{2-j}} z_{k, j}(\xi, \rho) d \xi \\
& -\frac{1}{4 \rho} \sum_{\alpha=k+1}^{4} \omega_{\alpha}^{s+1} \int_{x}^{1} e^{\rho\left(\omega_{\alpha}-\omega_{k}\right)(x-\xi)} \sum_{j=1}^{2} \frac{p_{j}(\xi)}{\rho^{2-j}} z_{k, j}(\xi, \rho) d \xi+O\left(\rho^{-3}\right),
\end{aligned}
$$

and particularly

$$
z_{k, s}(x, \rho)=\omega_{k}^{s}\left\{1-\frac{1}{4 \rho \omega_{k}} \int_{0}^{x} p_{2}(\xi) d \xi+O\left(\rho^{-2}\right)\right\} .
$$

From (22), the formulae

$$
z_{k, s}(0, \rho)=\omega_{k}^{s}-\frac{1}{4 \rho} \sum_{\alpha=k+1}^{4} \omega_{\alpha}^{s+1} B_{\alpha, k}(\rho)+O\left(\rho^{-3}\right)
$$


and

$$
\begin{aligned}
z_{k, s}(1, \rho)= & \omega_{k}^{s}+\frac{\omega_{k}^{s+1}}{4 \rho} \sum_{j=1}^{2} \frac{1}{\rho^{2-j}} \int_{0}^{1} p_{j}(\xi) z_{k, j}(\xi, \rho) d \xi \\
& +\frac{1}{4 \rho} \sum_{\alpha=1}^{k-1} \omega_{\alpha}^{s+1} B_{\alpha, k}(\rho)+O\left(\rho^{-3}\right)
\end{aligned}
$$

are valid, where

$B_{\alpha, k}(\rho)=\left\{\begin{array}{l}\sum_{j=1}^{2} \frac{1}{\rho^{2-j}} \int_{0}^{1} p_{j}(\xi) z_{k, j}(\xi, \rho) e^{-\rho\left(\omega_{\alpha}-\omega_{k}\right) \xi} d \xi, \quad \text { if } 1 \leq k<\alpha \leq 4, \\ \sum_{j=1}^{2} \frac{1}{\rho^{2-j}} \int_{0}^{1} p_{j}(\xi) z_{k, j}(\xi, \rho) e^{\rho\left(\omega_{\alpha}-\omega_{k}\right)(1-\xi)} d \xi, \text { if } 1 \leq \alpha<k \leq 4\end{array}\right.$

One can easily see that

$$
B_{\alpha, k}(\rho)=O\left(\rho^{-1}\right) .
$$

According to (3) and (20),

$$
\int_{0}^{1} p_{1}(\xi) z_{k, 1}(\xi, \rho) d \xi=\omega_{k} \int_{0}^{1} p_{1}(\xi) d \xi+O\left(\rho^{-1}\right)=c_{1} \omega_{k}+O\left(\rho^{-1}\right)
$$

and, by (23), the formula

$$
\begin{aligned}
& \int_{0}^{1} p_{2}(\xi) z_{k, 2}(\xi, \rho) d \xi \\
& \quad=\omega_{k}^{2} \int_{0}^{1} p_{2}(\xi)\left\{1-\frac{1}{4 \rho \omega_{k}} \int_{0}^{\xi} p_{2}(t) d t\right\} d \xi+O\left(\rho^{-2}\right)=O\left(\rho^{-2}\right)
\end{aligned}
$$

can be easily obtained. This two equalities require

$$
\frac{\omega_{k}^{s+1}}{4 \rho} \sum_{j=1}^{2} \frac{1}{\rho^{2-j}} \int_{0}^{1} p_{j}(\xi) z_{k, j}(\xi, \rho) d \xi=\frac{c_{1} \omega_{k}^{s+2}}{4 \rho^{2}}+O\left(\rho^{-3}\right) .
$$

From here, (25) can be rewritten as follows:

$$
z_{k, s}(1, \rho)=\omega_{k}^{s}+\frac{c_{1} \omega_{k}^{s+2}}{4 \rho^{2}}+\frac{1}{4 \rho} \sum_{\alpha=1}^{k-1} \omega_{\alpha}^{s+1} B_{\alpha, k}(\rho)+O\left(\rho^{-3}\right) .
$$

By using formulae (24) and (28), for $s=\overline{0,3}$, we obtain the following equalities: 


$$
\begin{aligned}
& z_{2, s}(0, \rho)=\omega_{2}^{s}-\frac{\omega_{3}^{s+1}}{4 \rho} B_{3,2}(\rho)-\frac{\omega_{4}^{s+1}}{4 \rho} B_{4,2}(\rho)+O\left(\rho^{-3}\right), \\
& z_{3, s}(0, \rho)=\omega_{3}^{s}-\frac{\omega_{4}^{s+1}}{4 \rho} B_{4,3}(\rho)+O\left(\rho^{-3}\right), \\
& z_{2, s}(1, \rho)=\omega_{2}^{s}+\frac{c_{1} \omega_{2}^{s+2}}{4 \rho^{2}}+\frac{\omega_{1}^{s+1}}{4 \rho} B_{1,2}(\rho)+O\left(\rho^{-3}\right), \\
& z_{3, s}(1, \rho)=\omega_{3}^{s}+\frac{c_{1} \omega_{3}^{s+2}}{4 \rho^{2}}+\frac{\omega_{1}^{s+1}}{4 \rho} B_{1,3}(\rho)+\frac{\omega_{2}^{s+1}}{4 \rho} B_{2,3}(\rho)+O\left(\rho^{-3}\right) .
\end{aligned}
$$

\section{Proof of Theorem 1}

Let

$$
\Delta(\rho)=\left|\begin{array}{llll}
U_{3}\left(y_{1}\right) & U_{3}\left(y_{2}\right) & U_{3}\left(y_{3}\right) & U_{3}\left(y_{4}\right) \\
U_{2}\left(y_{1}\right) & U_{2}\left(y_{2}\right) & U_{2}\left(y_{3}\right) & U_{2}\left(y_{4}\right) \\
U_{1}\left(y_{1}\right) & U_{1}\left(y_{2}\right) & U_{1}\left(y_{3}\right) & U_{1}\left(y_{4}\right) \\
U_{0}\left(y_{1}\right) & U_{0}\left(y_{2}\right) & U_{0}\left(y_{3}\right) & U_{0}\left(y_{4}\right)
\end{array}\right|,
$$

where $y_{k}(x, \rho), k=\overline{1,4}$, are the linearly independent solutions of the Eq. (16). If we properly choose the vertex $\rho=-c$ of the domain $T_{0}$, then the eigenvalues $\lambda$ of the differential operator (1)-(2) whose absolute values are sufficiently large have the form $\lambda=-\rho^{4}$, where the numbers $\rho$ are the roots of the equation

$$
\Delta(\rho)=0
$$

in the domain $T_{0}$, and the set of such points $\rho$ includes all the roots of (31) in the domain $T_{0}$ except for a finite number (see [30, Chapter II. $\left.\S 4.9\right]$ ). By (19), for $s=\overline{0,3}$ and $k=\overline{1,4}$, we have

$$
U_{s}\left(y_{k}\right)=\rho^{s}\left\{e^{\rho \omega_{k}} z_{k, s}(1, \rho)-(-1)^{\sigma} z_{k, s}(0, \rho)\right\} .
$$

According to (15), $e^{\rho \omega_{1}}$ tends exponentially to zero and $e^{\rho \omega_{4}}$ tends exponentially to infinity. Consequently, the following equalities are valid by (20) and (32):

$$
\begin{aligned}
& U_{s}\left(y_{1}\right)=-\rho^{s}(-1)^{\sigma}\left\{z_{1, s}(0, \rho)+O\left(\rho^{-1}\right)\right\} \\
& U_{s}\left(y_{4}\right)=\rho^{s} e^{\rho \omega_{4}}\left\{z_{4, s}(1, \rho)+O\left(\rho^{-1}\right)\right\}
\end{aligned}
$$

i.e.

$$
\begin{aligned}
& U_{s}\left(y_{1}\right)=-\rho^{s}(-1)^{\sigma}\left\{\omega_{1}^{s}+O\left(\rho^{-1}\right)\right\}, \\
& U_{s}\left(y_{4}\right)=\rho^{s} e^{\rho \omega_{4}}\left\{\omega_{4}^{s}+O\left(\rho^{-1}\right)\right\} .
\end{aligned}
$$

Let

$$
A_{s, k}(\rho)=e^{\rho \omega_{k}} z_{k, s}(1, \rho)-(-1)^{\sigma} z_{k, s}(0, \rho), \quad k=2,3, \quad s=\overline{0,3} .
$$


Due to (32), it is obvious that

$$
U_{s}\left(y_{k}\right)=\rho^{s} A_{s, k}(\rho), \quad k=2,3, \quad s=\overline{0,3} .
$$

By using the formulae (64) and (65) in [15], if $\rho$ is a root of Eq. (31), we get that the following equalities are valid:

$$
e^{\rho \omega_{k}}-(-1)^{\sigma}=O\left(\rho^{-2}\right), \quad k=2,3
$$

because $p_{2}(x) \in W_{1}^{1}(0,1)$ and $\alpha_{1,0}=\alpha_{2,1}=\alpha_{3,2}=0$ by present conditions. From here, (27) and (29), we get

$$
A_{s, k}(\rho)=C_{s, k}(\rho)+D_{s, k}(\rho)+O\left(\rho^{-3}\right), \quad k=2,3, \quad s=\overline{0,3}
$$

and

$$
A_{s, k}(\rho)=O\left(\rho^{-2}\right), \quad k=2,3, \quad s=\overline{0,3},
$$

where

$$
\begin{aligned}
C_{s, k}(\rho) & =\omega_{k}^{s}\left(e^{\rho \omega_{k}}-(-1)^{\sigma}\right)+\frac{(-1)^{\sigma} c_{1} \omega_{k}^{s+2}}{4 \rho^{2}}+\frac{(-1)^{\sigma} \omega_{5-k}^{s+1}}{4 \rho} B_{5-k, k}(\rho), \\
D_{s, k}(\rho) & =\frac{(-1)^{\sigma} \omega_{1}^{s+1}}{4 \rho} B_{1, k}(\rho)+\frac{(-1)^{\sigma} \omega_{4}^{s+1}}{4 \rho} B_{4, k}(\rho) \\
k & =2,3, \quad s=\overline{0,3} .
\end{aligned}
$$

We substitute these expressions in the Eq. (31) (see (30)) and divide out the common factors $\rho^{3}, \rho^{2}, \rho$ of the rows and also the common factor $-(-1)^{\sigma}$ and $e^{\rho \omega_{4}}$ of the first and last column of the determinant $\Delta(\rho)$. Then the Eq. (31) can be written in the form

$$
\Delta^{(1)}(\rho)+O\left(\rho^{-5}\right)=0
$$

where

$$
\Delta^{(1)}(\rho)=\left|\begin{array}{cccc}
\omega_{1}^{3} & A_{3,2}(\rho) & A_{3,3}(\rho) & \omega_{4}^{3} \\
\omega_{1}^{2} & A_{2,2}(\rho) & A_{2,3}(\rho) & \omega_{4}^{2} \\
\omega_{1} & A_{1,2}(\rho) & A_{1,3}(\rho) & \omega_{4} \\
1 & A_{0,2}(\rho) & A_{0,3}(\rho) & 1
\end{array}\right| .
$$

Taken into account (37) in the determinant (41) (see (39)), one can easily see that the column vectors $D_{s, k}(\rho)$ for $k=2,3$ are linear combinations of first and last columns. As a result, (40) is reduced to the following equation:

$$
\Delta^{(2)}(\rho)+O\left(\rho^{-5}\right)=0
$$

where

$$
\Delta^{(2)}(\rho)=\left|\begin{array}{cccc}
\omega_{1}^{3} & C_{3,2}(\rho) & C_{3,3}(\rho) & \omega_{4}^{3} \\
\omega_{1}^{2} & C_{2,2}(\rho) & C_{2,3}(\rho) & \omega_{4}^{2} \\
\omega_{1} & C_{1,2}(\rho) & C_{1,3}(\rho) & \omega_{4} \\
1 & C_{0,2}(\rho) & C_{0,3}(\rho) & 1
\end{array}\right|
$$


After calculating the determinant (43), by (11) and (39), the Eq. (42) is equivalent to

$$
\begin{aligned}
& -16\left(e^{\rho \omega_{2}}-(-1)^{\sigma}\right)\left(e^{\rho \omega_{3}}-(-1)^{\sigma}\right)-\frac{4(-1)^{\sigma} i c_{1}\left(e^{\rho \omega_{2}}-(-1)^{\sigma}\right)}{\rho^{2}} \\
& -\frac{4(-1)^{\sigma} i c_{1}\left(e^{\rho \omega_{3}}-(-1)^{\sigma}\right)}{\rho^{2}}+\frac{c_{1}^{2}}{\rho^{4}}-\frac{i B_{3,2}(\rho) B_{2,3}(\rho)}{\rho^{2}}+O\left(\rho^{-5}\right)=0 .
\end{aligned}
$$

From Riemann-Lebesque Lemma, for an arbitrary function $f(x) \in$ $L_{1}(0,1)$, the formulae

$$
\int_{0}^{1} f(\xi) e^{\tau \xi} d \xi=o(1), \quad \int_{0}^{1} f(\xi) e^{\tau(1-\xi)} d \xi=o(1), \quad|\tau| \rightarrow+\infty
$$

hold for $\Re \tau \leq c_{1}=$ const. Using (12), (20), (26), (36), (45) and integration by parts, we have

$$
B_{3,2}(\rho) B_{2,3}(\rho)=-\frac{i\left(p_{2}(1)-p_{2}(0)\right)^{2}}{4 \rho^{2}}+O\left(\rho^{-2} \varepsilon(\rho)\right),
$$

where

$$
\begin{aligned}
\varepsilon(\rho)= & \left|\int_{0}^{1}\left(2 p_{1}(\xi)-p_{2}^{\prime}(\xi)\right) e^{2 \rho \omega_{2} \xi} d \xi\right| \\
& +\left|\int_{0}^{1}\left(2 p_{1}(\xi)-p_{2}^{\prime}(\xi)\right) e^{2 \rho \omega_{2}(1-\xi)} d \xi\right|+\left|\rho^{-1}\right| .
\end{aligned}
$$

Also, $\varepsilon(\rho)$ satisfies

$$
\varepsilon(\rho)=o(1)
$$

by (45).

From (46), we can rewrite the Eq. (44) as follows:

$$
\begin{aligned}
& -16\left(e^{\rho \omega_{2}}-(-1)^{\sigma}\right)\left(e^{\rho \omega_{3}}-(-1)^{\sigma}\right)-\frac{4(-1)^{\sigma} i c_{1}\left(e^{\rho \omega_{2}}-(-1)^{\sigma}\right)}{\rho^{2}} \\
& -\frac{4(-1)^{\sigma} i c_{1}\left(e^{\rho \omega_{3}}-(-1)^{\sigma}\right)}{\rho^{2}}+\frac{c_{1}^{2}}{\rho^{4}}-\frac{\left(p_{2}(1)-p_{2}(0)\right)^{2}}{4 \rho^{4}}+O\left(\rho^{-4} \varepsilon(\rho)\right)=0 .
\end{aligned}
$$

After some simplifications, the equation above is reduced to the form

$$
\begin{aligned}
& \left(16-\frac{4 i c_{1}}{\rho^{2}}\right) e^{2 \rho \omega_{2}}-2(-1)^{\sigma}\left(16-\frac{4 i c_{1}}{\rho^{2}}-\frac{c_{1}^{2}}{2 \rho^{4}}+\frac{\left(p_{2}(1)-p_{2}(0)\right)^{2}}{8 \rho^{4}}\right) e^{\rho \omega_{2}} \\
& +\left(16-\frac{4 i c_{1}}{\rho^{2}}\right)+O\left(\rho^{-4} \varepsilon(\rho)\right)=0 .
\end{aligned}
$$


The last equation splits into two equations:

$$
\begin{aligned}
& e^{\rho \omega_{2}}=(-1)^{\sigma}-\frac{\gamma_{0}}{8 \rho^{2}}+O\left(\rho^{-2} \varepsilon(\rho)\right), \\
& e^{\rho \omega_{2}}=(-1)^{\sigma}+\frac{\gamma_{0}}{8 \rho^{2}}+O\left(\rho^{-2} \varepsilon(\rho)\right),
\end{aligned}
$$

where $\gamma_{0}$ is the number defined by (4).

Now, we will investigate the Eq. (48). By using Rouche's theorem, it can be proved in a standard way that the roots $\rho \in T_{0}$ of the Eq. (48) whose absolute values are sufficiently large lie on the domains $G_{n} \subset T_{0}, n=n_{0}, n_{0}+$ $1, \ldots$, where $G_{n}$ is the $O\left(n^{-1}\right)$-neighborhood of the point $-(2 n-\sigma) \pi i / \omega_{2}$ and $n_{0}$ is sufficiently large natural number (see [30, Chapter II, $\left.\S 4.9\right]$ ). Moreover, (48) has a unique root within each $G_{n}, n=n_{0}, n_{0}+1, \ldots$ Let $\widetilde{\rho}$ be the root of the Eq. (48) belonging to $G_{n}$. By using the equalities (67) and (71) from [15], we have

$$
\widetilde{\rho}=-\frac{(2 n-\sigma) \pi i}{\omega_{2}}+r, \quad r=O\left(n^{-2}\right) .
$$

From here, the equalities

$$
\begin{aligned}
& \frac{1}{\widetilde{\rho}}=\frac{-\omega_{2}}{(2 n-\sigma) \pi i}+O\left(n^{-4}\right), \quad \frac{1}{\widetilde{\rho}^{2}}=\frac{1}{(2 n-\sigma)^{2} \pi^{2} i}+O\left(n^{-5}\right), \\
& e^{\tilde{\rho} \omega_{2}}=(-1)^{\sigma}\left\{1+r \omega_{2}+O\left(n^{-4}\right)\right\}
\end{aligned}
$$

can be easily obtained. By (7), (47), (50) and (51), we calculate as

$$
\varepsilon(\rho)=O\left(\varepsilon_{n}\right) .
$$

Writing $\rho=\widetilde{\rho}$ in (48) and using the relations (51) and (52), after simple transformations we have

$$
r=\frac{-(-1)^{\sigma} \gamma_{0}}{8 \omega_{2}(2 n-\sigma)^{2} \pi^{2} i}+O\left(n^{-2} \varepsilon_{n}\right) .
$$

Thus, by (50) and (53), within $O\left(n^{-1}\right)$-neighborhood $G_{n}$ of the point $z_{n}=-(2 n-\sigma) \pi i / \omega_{2}, n=n_{0}, n_{0}+1, \ldots$, the Eq. (48) has the unique root

$$
\widetilde{\rho}_{n, 1}=-\frac{1}{\omega_{2}}\left\{(2 n-\sigma) \pi i+\frac{\gamma_{0}}{8(2 n-\sigma)^{2} \pi^{2} i}\right\}+O\left(n^{-2} \varepsilon_{n}\right) .
$$

Similarly, we find that, within $O\left(n^{-1}\right)$-neighborhood $G_{n}$ of the point $z_{n}$, $n=n_{0}, n_{0}+1, \ldots$, the Eq. (49) has the unique root

$$
\widetilde{\rho}_{n, 2}=-\frac{1}{\omega_{2}}\left\{(2 n-\sigma) \pi i-\frac{\gamma_{0}}{8(2 n-\sigma)^{2} \pi^{2} i}\right\}+O\left(n^{-2} \varepsilon_{n}\right) .
$$


We seek the eigenfunction $\widetilde{u}_{n, j}(x), j=1,2$, corresponding to the eigenvalue $\lambda=-\left(\widetilde{\rho}_{n, j}\right)^{4}, j=1,2$, for sufficiently large $n$, in the form

$$
\widetilde{u}_{n, j}(x)=\frac{i e^{-\rho \omega_{4}}}{\rho^{4}}\left|\begin{array}{cccc}
y_{1}(x, \rho) & y_{2}(x, \rho) & y_{3}(x, \rho) & y_{4}(x, \rho) \\
U_{3}\left(y_{1}\right) & U_{3}\left(y_{2}\right) & U_{3}\left(y_{3}\right) & U_{3}\left(y_{4}\right) \\
U_{2}\left(y_{1}\right) & U_{2}\left(y_{2}\right) & U_{2}\left(y_{3}\right) & U_{2}\left(y_{4}\right) \\
U_{1}\left(y_{1}\right) & U_{1}\left(y_{2}\right) & U_{1}\left(y_{3}\right) & U_{1}\left(y_{4}\right)
\end{array}\right|,
$$

or, more precisely

$$
\begin{aligned}
\widetilde{u}_{n, j}(x)= & -(-1)^{\sigma} i \rho^{2} \\
& \times\left|\begin{array}{cccc}
-(-1)^{\sigma} y_{1}(x, \rho) & y_{2}(x, \rho) & y_{3}(x, \rho) & e^{-\rho \omega_{4}} y_{4}(x, \rho) \\
-(-1)^{\sigma} \rho^{-3} U_{3}\left(y_{1}\right) & \rho^{-3} U_{3}\left(y_{2}\right) & \rho^{-3} U_{3}\left(y_{3}\right) & \rho^{-3} e^{-\rho \omega_{4}} U_{3}\left(y_{4}\right) \\
-(-1)^{\sigma} \rho^{-2} U_{2}\left(y_{1}\right) & \rho^{-2} U_{2}\left(y_{2}\right) & \rho^{-2} U_{2}\left(y_{3}\right) & \rho^{-2} e^{-\rho \omega_{4}} U_{2}\left(y_{4}\right) \\
-(-1)^{\sigma} \rho^{-1} U_{1}\left(y_{1}\right) & \rho^{-1} U_{1}\left(y_{2}\right) & \rho^{-1} U_{1}\left(y_{3}\right) & \rho^{-1} e^{-\rho \omega_{4}} U_{1}\left(y_{4}\right)
\end{array}\right|,
\end{aligned}
$$

where $\rho=\widetilde{\rho}_{n, j}$.

With (35), we can write

$$
\begin{aligned}
\widetilde{u}_{n, j}(x)=- & (-1)^{\sigma} i \rho^{2} \\
\times & \left|\begin{array}{cccc}
-(-1)^{\sigma} y_{1}(x, \rho) & y_{2}(x, \rho) & y_{3}(x, \rho) & e^{-\rho \omega_{4}} y_{4}(x, \rho) \\
-(-1)^{\sigma} \rho^{-3} U_{3}\left(y_{1}\right) & A_{3,2}(\rho) & A_{3,3}(\rho) & \rho^{-3} e^{-\rho \omega_{4}} U_{3}\left(y_{4}\right) \\
-(-1)^{\sigma} \rho^{-2} U_{2}\left(y_{1}\right) & A_{2,2}(\rho) & A_{2,3}(\rho) & \rho^{-2} e^{-\rho \omega_{4}} U_{2}\left(y_{4}\right) \\
-(-1)^{\sigma} \rho^{-1} U_{1}\left(y_{1}\right) & A_{1,2}(\rho) & A_{1,3}(\rho) & \rho^{-1} e^{-\rho \omega_{4}} U_{1}\left(y_{4}\right)
\end{array}\right|_{\rho=\widetilde{\rho}_{n, j}} .
\end{aligned}
$$

From (19) and (20), we get

$$
y_{k}(x, \rho)=O(1), \quad k=1,2,3, \quad e^{-\rho \omega_{4}} y_{4}(x, \rho)=O(1) .
$$

In view of (33), (38), (56) and (57), we have

$$
\widetilde{u}_{n, j}(x)=-(-1)^{\sigma} i \rho^{2}\left\{y_{3}(x, \rho) E_{2}(\rho)-y_{2}(x, \rho) E_{3}(\rho)\right\}+O\left(\rho^{-1}\right) \text {, }
$$

where $\rho=\widetilde{\rho}_{n, j}$ and

$$
E_{k}(\rho)=\left|\begin{array}{lll}
\omega_{1}^{3} & A_{3, k}(\rho) & \omega_{4}^{3} \\
\omega_{1}^{2} & A_{2, k}(\rho) & \omega_{4}^{2} \\
\omega_{1} & A_{1, k}(\rho) & \omega_{4}
\end{array}\right|_{\rho=\widetilde{\rho}_{n, j}}, \quad k=2,3
$$

Using (37) and (39), we reduce the determinant $E_{k}(\rho)$ to

$$
E_{k}(\rho)=\left|\begin{array}{lll}
\omega_{1}^{3} & C_{3, k}(\rho) & \omega_{4}^{3} \\
\omega_{1}^{2} & C_{2, k}(\rho) & \omega_{4}^{2} \\
\omega_{1} & C_{1, k}(\rho) & \omega_{4}
\end{array}\right|_{\rho=\widetilde{\rho}_{n, j}}+O\left(\rho^{-3}\right), \quad k=2,3
$$


If we calculate this determinant using (48) and (49), we will get

$$
E_{k}(\rho)=\frac{(-1)^{j} \gamma_{0}+(-1)^{k+\sigma} i\left(2 c_{1}+\left(p_{2}(1)-p_{2}(0)\right)\right)}{2 \rho^{2}}+O\left(\rho^{-2} \varepsilon(\rho)\right),
$$

where $k=2,3$ and $\rho=\widetilde{\rho}_{n, j}$. By (19), (20), (54) and (55), we have

$$
\begin{aligned}
y_{2}\left(x, \widetilde{\rho}_{n, j}\right) & =e^{-(2 n-\sigma) \pi i x}+O\left(n^{-2}\right), \quad y_{3}\left(x, \widetilde{\rho}_{n, j}\right)=e^{(2 n-\sigma) \pi i x}+O\left(n^{-2}\right), \\
\left(\widetilde{\rho}_{n, j}\right)^{-1} & =O\left(n^{-1}\right),
\end{aligned}
$$

where $j=1,2$. By using these expressions and (52), (57), (58), (59) we have the representation for $j=1,2$,

$$
\begin{aligned}
\widetilde{u}_{n, j}(x)= & \left(2 c_{1}+\left(p_{2}(1)-p_{2}(0)\right)\right) \cos (2 n-\sigma) \pi x \\
& (-1)^{j} \gamma_{0} \sin (2 n-\sigma) \pi x+O\left(\varepsilon_{n}\right) .
\end{aligned}
$$

The construction of the integers $n_{1}$ and $n_{2}$ is completely similar to the method in [13-15]. Consequently, the formulae (5) and (6) are directly obtained from (54), (55), (60) and the relation $\lambda=-\rho^{4}$.

\section{Proofs of Theorem 2 and Corollary 1}

Since $p_{j}(x) \in W_{1}^{j}(0,1), j=1,2$, by $(7)$, we have $\varepsilon_{n}=O\left(n^{-1}\right)$. Consequently, in this case, the formula (8) are true.

Let

$$
v_{1,1}(x), v_{1,2}(x), \ldots, v_{n, 1}(x), v_{n, 2}(x), \ldots
$$

be the system which is biorthogonally conjugate to the system

$$
u_{1,1}(x), u_{1,2}(x), \ldots, u_{n, 1}(x), u_{n, 2}(x), \ldots,
$$

i.e. $\left(u_{n, j}, v_{m, s}\right)=\delta_{n, m} . \delta_{j, s}, n, m=1,2, \ldots, j, s=1,2$. It is well known from $[17$, p.84] or $[30$, p.99] that $(61)$ is the system of root functions of the differential operator $L^{*}$ which is the adjoint operator to $L$. By the conditions $p_{2}^{(s)}(1)=$ $p_{2}^{(s)}(0), s=0,1$ and $p_{1}(1)=p_{1}(0)$ of Theorem 2 , the differential operator $L^{*}$ is generated by the following differential expression and adjoint boundary conditions

$$
\begin{aligned}
& l^{*}(z)=z^{\mathrm{iv}}+\left(\overline{p_{2}(x)} z\right)^{\prime \prime}-\left(\overline{p_{1}(x)} z\right)^{\prime}+\overline{p_{0}(x)} z \\
& U_{0}^{*}(z) \equiv z(1)-(-1)^{\sigma} z(0)=0 \\
& U_{1}^{*}(z) \equiv z^{\prime}(1)-(-1)^{\sigma} z^{\prime}(0)=0 \\
& U_{2}^{*}(z) \equiv z^{\prime \prime}(1)-(-1)^{\sigma} z^{\prime \prime}(0)=0 \\
& U_{3}^{*}(z) \equiv z^{\prime \prime \prime}(1)-(-1)^{\sigma} z^{\prime \prime \prime}(0)=0 .
\end{aligned}
$$


From the form of the differential operator $L^{*}$ and Theorem 1, it follows that for sufficiently large numbers $n$ the equality

$$
\begin{aligned}
& \overline{v_{n+n_{j}, j}(x)} \\
& \quad=r_{n+n_{j}, j}\left(\cos (2 n-\sigma) \pi x+(-1)^{j}(-i)^{1-\sigma} \sin (2 n-\sigma) \pi x+O\left(n^{-1}\right)\right),
\end{aligned}
$$

is valid, where $r_{n_{j}+n, j}, j=1,2$ are some numbers determined from the equality $\left(u_{n_{j}+n, j}, v_{n_{j}+n, j}\right)=1, j=1,2$. According to the equality $\left(u_{n+n_{j}, j}, v_{n+n_{j}, j}\right)=$ $1, j=1,2$, and the asymptotic formulae (8), (64), for sufficiently large numbers $n$, we have $r_{n+n_{j}, j}=1+O\left(n^{-1}\right), j=1,2$. Consequently, from (64), for sufficiently large numbers $n$, we obtain

$$
\overline{v_{n+n_{j}, j}(x)}=\cos (2 n-\sigma) \pi x+(-1)^{j}(-i)^{1-\sigma} \sin (2 n-\sigma) \pi x+O\left(n^{-1}\right) .
$$

Let

$$
\begin{aligned}
& g_{0}(x)=1, \quad g_{2 n-1}(x)=\sqrt{2} \sin 2 n \pi x, \quad g_{2 n}(x)=\sqrt{2} \cos 2 n \pi x, \\
& \widetilde{g}_{2 n-1}=\sqrt{2} \sin (2 n-1) \pi x, \quad \widetilde{g}_{2 n}=\sqrt{2} \cos (2 n-1) \pi x, \\
& h_{0}(x)=1, \quad h_{2(n-1)+j}(x)=\cos 2 n \pi x+(-1)^{j} i \sin 2 n \pi x, \\
& \widetilde{h}_{2(n-1)+j}(x)=\cos (2 n-1) \pi x+(-1)^{j} \sin (2 n-1) \pi x,
\end{aligned}
$$

where $n=1,2, \ldots, j=1,2$. Each of the systems (66) and (67) is an orthonormal basis of the space $L_{2}(0,1)$. From the asymptotic formulae (8), (65), it is obvious that each of the systems (61) and (62) satisfies the Bessel inequality. Namely, for an arbitrary function $f(x) \in L_{2}(0,1)$,

$$
\sum_{n=1}^{\infty} \sum_{j=1}^{2}\left|\left(f, u_{n, j}\right)\right|^{2}<+\infty, \quad \sum_{n=1}^{\infty} \sum_{j=1}^{2}\left|\left(f, v_{n, j}\right)\right|^{2}<+\infty .
$$

Furthermore, each of the systems (61) and (62) is complete in the space $L_{2}(0,1)$ (see [2]). Consequently each of these systems forms a Riesz basis of the space $L_{2}(0,1)$ (see [10, Chapter VI, $\S 2.2$ Theorem 2.1]).

Let us prove Corollary 1. According to Lemma 2 in [14], each of the systems (68) and (69) (see (66) and (67)) is a Riesz basis in the space $L_{2}(0,1)$. Consider the case $\sigma=0$. The case $\sigma=1$ can be checked in the same way by using (69). Assume that $n_{1} \geq 0$ and $n_{2} \geq 0$. From the asymptotic formulae (6) and the definition of $\left\{h_{k}(x)\right\}_{k=0}^{\infty}$ (see (68)), we obtain

$$
\sum_{n=1}^{\infty}\left(\left\|u_{n+n_{1}, 1}-h_{2 n-1}\right\|^{2}+\left\|u_{n+n_{2}, 2}-h_{2 n}\right\|^{2}\right) \leq \mathrm{const} \sum_{n=1}^{\infty} \frac{1}{n^{2}}<+\infty .
$$

It is easy to see that $n_{1}+n_{2}$ root functions of the operator $L$ and one function from system (68) are absent in (70). Let $n_{1}+n_{2}>1$. In this case, by (70), 
the system $S$ generated by all functions except $n_{1}+n_{2}-1$ functions from the system (62) is quadratically close to system (68). Since (68) is a Riesz basis of $L_{2}(0,1)$, then $S$ also forms a Riesz basis of $L_{2}(0,1)[10]$. The latter contradicts the basicity of the system $(62)$ in $L_{2}(0,1)$.

Let $n_{1}=n_{2}=0$. Since (62) is a Riesz basis of the space $L_{2}(0,1)$, then again by (70), the system $\left\{h_{k}(x)\right\}_{k=1}^{\infty}$ is a basis of the space $L_{2}(0,1)$ and this contradicts the basicity of the system $\left\{h_{k}(x)\right\}_{k=0}^{\infty}$ in $L_{2}(0,1)$. All the remaining cases can be investigated in a similar way.

Thus, $n_{1}+n_{2}=1$ holds. Therefore, without loss of generality we can assume that $n_{1}=0, n_{2}=1-\sigma$. Consequently, from (8) and (65), we have

$$
\begin{aligned}
& u_{n, 1}(x)=\cos (2 n-\sigma) \pi x-i^{1-\sigma} \sin (2 n-\sigma) \pi x+O\left(n^{-1}\right), \\
& u_{n+1-\sigma, 2}(x)=\cos (2 n-\sigma) \pi x+i^{1-\sigma} \sin (2 n-\sigma) \pi x+O\left(n^{-1}\right), \\
& \overline{v_{n, 1}(x)}=\cos (2 n-\sigma) \pi x-(-i)^{1-\sigma} \sin (2 n-\sigma) \pi x+O\left(n^{-1}\right), \\
& \overline{v_{n+1-\sigma, 2}(x)}=\cos (2 n-\sigma) \pi x+(-i)^{1-\sigma} \sin (2 n-\sigma) \pi x+O\left(n^{-1}\right) .
\end{aligned}
$$

Next we prove that the system of the root functions of the differential operator $L$ forms a basis of the space $L_{p}(0,1)$, where $1<p<\infty$ and $p \neq 2$. As above, we consider only the case $\sigma=0$. The case $\sigma=1$ is similar. Note that $(66)$ is a basis of the space $L_{p}(0,1)$ for any $p \in(1, \infty)$ [1, Chapter VIII, $\S 20$, Theorem 2]. Consequently, there exists a constant $M_{p}>0$ ensuring the inequality

$$
\left\|\sum_{n=0}^{N}\left(f, g_{n}\right) g_{n}\right\|_{p} \leq M_{p}\|f\|_{p}, \quad N=1,2, \ldots,
$$

for any function $f(x) \in L_{p}(0,1)$, where $\|\cdot\|_{p}$ means the norm in the space $L_{p}(0,1)$ (see $[12$, Chapter I, $\S 4$, Theorem 6$]$ ). We now fix $p \in(1,2)$. Since the system (62) is complete in the space $L_{2}(0,1)$, then this system is complete in $L_{p}(0,1)$ as well. Moreover, it is easy to see that

$$
\left\|\left(f, v_{n, j}\right) u_{n, j}\right\|_{p} \leq \mathrm{const}\|f\|_{p},
$$

where $n=1,2, \ldots$ and $j=1,2$. Consequently, in order to prove the basicity of this system in $L_{p}(0,1)$, it is enough to prove the existence of a constant $M>0$ ensuring the inequality

$$
\left\|\sum_{n=1}^{m} \sum_{j=1}^{2}\left(f, v_{n, j}\right) u_{n, j}\right\|_{p} \leq M\|f\|_{p} \quad m=1,2, \ldots,
$$

for $f(x) \in L_{p}(0,1)$ (see [12, Chapter VIII, $\S 4$, Theorem 6]). Note that instead of this inequality, under the same conditions, it is enough to prove the inequality 


$$
J_{m}(f)=\left\|\sum_{n=1}^{m}\left\{\left(f, v_{n, 1}\right) u_{n, 1}+\left(f, v_{n+1,2}\right) u_{n+1,2}\right\}\right\|_{p} \leq M^{\prime}\|f\|_{p},
$$

where $m=1,2, \ldots$ and $M^{\prime}$ is a certain positive constant.

From (66) and (71), we have

$$
J_{m}(f) \leq J_{m, 1}(f)+J_{m, 2}(f)+J_{m, 3}(f)+J_{m, 4}(f),
$$

where $m=1,2, \ldots$ and

$$
\begin{aligned}
& J_{m, 1}(f)=\left\|\sum_{n=1}^{2 m}\left(f, g_{n}\right) g_{n}\right\|_{p}, \quad J_{m, 2}(f)=\left\|\sum_{n=1}^{2 m}\left(f, g_{n}\right) O\left(n^{-1}\right)\right\|_{p}, \\
& J_{m, 3}(f)=\left\|\sum_{n=1}^{2 m}\left(f, O\left(n^{-1}\right)\right) g_{n}\right\|_{p}, \quad J_{m, 4}(f)=\left\|\sum_{n=1}^{2 m}\left(f, O\left(n^{-1}\right)\right) O\left(n^{-1}\right)\right\|_{p} .
\end{aligned}
$$

By (72),

$$
J_{m, 1}(f) \leq \text { const }\|f\|_{p} .
$$

By Riesz theorem, it follows that

$$
\begin{aligned}
J_{m, 2}(f) & \leq \text { const } \sum_{n=1}^{2 m}\left|\left(f, g_{n}\right)\right| n^{-1} \\
& \leq \text { const }\left(\sum_{n=1}^{2 m}\left|\left(f, g_{n}\right)\right|^{q}\right)^{1 / q}\left(\sum_{n=1}^{2 m} n^{-p}\right)^{1 / p} \leq \text { const }\|f\|_{p},
\end{aligned}
$$

where $1 / p+1 / q=1$ (see [36, Chapter XII, $\S 2$, Theorem 2.8]). Further,

$$
\begin{aligned}
& J_{m, 3}(f) \leq\left\|\sum_{n=1}^{2 m}\left(f, O\left(n^{-1}\right)\right) g_{n}\right\|_{2}=\left(\sum_{n=1}^{2 m}\left|\left(f, O\left(n^{-1}\right)\right)\right|^{2}\right)^{1 / 2} \\
& \text { const }\|f\|_{1}\left(\sum_{n=1}^{2 m} n^{-2}\right)^{1 / 2} \leq \mathrm{const}\|f\|_{p} .
\end{aligned}
$$

Moreover,

$$
J_{m, 4} \leq \text { const }\|f\|_{1} \sum_{n=1}^{2 m} n^{-2} \leq \text { const }\|f\|_{p} .
$$

The inequality (73) is a consequence of the inequalities (74)-(78). Thus, the basicity of the system $(62)$ in the space $L_{p}(0,1)$ for $1<p<2$ is proved.

Let $2<p<\infty$ and $1 / p+1 / q=1$. Note that $1<q<2$ and the system $(61)$ is the system of root functions of the differential operator $L^{*}$. As it has been proved above, the system of root functions of such operator forms a basis of the space $L_{r}(0,1)$ for any $r \in(1,2)$, in particular $r=q$. Thus, the system $(61)$ is a basis of $L_{q}(0,1)$. Consequently, the system (62) which is biorthogonally conjugate to (61) forms a basis of $L_{p}(0,1)$. 


\section{References}

[1] Bari, N.K.: Trigonometric Series, vol. 2. Macmillan, New York; Pergamon Press, Oxford (1964)

[2] Birkhoff, G.D.: Boundary value and expansion problems of ordinary linear differential equations. Trans. Am. Math. Soc. 9(4), 373-395 (1908)

[3] Dernek, N., Veliev, O.A.: On the Riesz basisness of the root functions of the nonself-adjoint Sturm-Liouville operator. Isr. J. Math. 145, 113-123 (2005)

[4] Djakov, P., Mityagin, B.: Convergence of spectral decompositions of Hill operators with trigonometric polynomial potentials. Doklady Math. 83, 5-7 (2011)

[5] Djakov, P., Mityagin, B.: Convergence of spectral decompositions of Hill operators with trigonometric polynomial potentials. Math. Ann. 351, 509-540 (2011)

[6] Djakov, P., Mityagin, B.: Criteria for existance of Riesz bases consisting of root functions of Hill and 1D Dirac operators. J. Funct. Anal. 263, 2300-2332 (2012)

[7] Djakov, P., Mityagin, B.: Instability zones one-dimesional periodic Shrödinger and Dirac operators (Russian). Uspehi Mat. Nauk. 61(4), 77-182 (2006) [English: Russian Math. Surveys 61(4), 663-766 (2006)]

[8] Dunford, N., Schwartz, J.T.: Linear Operators, Part III: Spectral Operators. Wiley-Interscience, New York (1988)

[9] Gesztesy, F., Tkachenko, V.: A Schauder and Riesz basis criterion for non-selfadjoint Schrodinger operators with periodic and antiperiodic boundary conditions. J. Differ. Equ. 253, 400-437 (2012)

[10] Gohberg, I.C., Krein M.G.: Introduction to the Theory of Linear Nonselfadjoint Operators. American Mathematical Society, Providence (1969)

[11] Ionkin, N.I.: Solutions of boundary value problem in heat conductions theory with nonlocal boundary conditions. Differ. Uravn. (in Russian) 13(2), 294304 (1977)

[12] Kashin, B.S., Saakyan, A.A.: Orthogonal Series. American Mathematical Society, Providence (1989)

[13] Kerimov, N.B., Kaya, U.: Some problems of spectral theory of fourth-order differential operators with regular boundary conditions. Arab. J. Math. 3(1), 49$61(2014)$

[14] Kerimov, N.B., Kaya, U.: Spectral asymptotics and basis properties of fourth order differential operators with regular boundary conditions. Math. Methods Appl. Sci. 37(5), 698-710 (2014)

[15] Kerimov, N.B., Kaya, U.: Spectral properties of some regular boundary value problems for fourth order differential operators. Central Eur. J. Math. 11(1), 94$111(2013)$

[16] Kerimov, N.B., Mamedov, Kh.R.: On the Riesz basis property of root functions of some regular boundary value problems. Math. Notes 64(4), 483-487 (1998)

[17] Keselman, G.M.: On the unconditional convergence of eigenfunction expansions of certain differential operators. Izv. Vyssh. Uchebn. Zaved. Mat. (in Russian) 39(2), 82-93 (1964)

[18] Kirac, A.A.: Riesz basis property of the root functions of non-selfadjoint operators with regular boundary conditions. Int. J. Math. Anal. 3, 1101-1109 (2009) 
[19] Makin, A.S.: Asymptotics of the spectrum of the Sturm-Liouville operator with regular boundary conditions. Differ. Equ. 44(5), 645-658 (2008)

[20] Makin, A.S.: Characterization of the spectrum of regular boundary value problems for the Sturm-Liouville operator. Differ. Equ. 44(3), 341-348 (2008)

[21] Makin, A.S.: Convergence of expansions in the root functions of periodic boundary value problems. Dokl. Math. 73(1), 71-76 (2006)

[22] Makin, A.S.: On a certain class of boundary-value problems for the SturmLiouville operator. Differents. Uravn. 35(8), 1058-1066 (1999)

[23] Makin, A.S.: On spectral decompositions corresponding to non-self-adjoint Sturm-Liouville operators. Dokl. Math. 73(1), 15-18 (2006)

[24] Makin, A.S.: On the basis property of systems of root functions of regular boundary value problems for the Sturm-Liouville operator. Differ. Equ. 42(12), 1717$1728(2006)$

[25] Mamedov, Kh.R., Menken, H.: On the basisness in $L_{2}(0,1)$ of the root functions in not strongly regular boundary value problems. Eur. J. Pure Appl. Math. 1(2), 51-60 (2008)

[26] Mamedov, Kh.R.: On the basis property in $L_{p}(0,1)$ of the root functions of a class non self adjoint Sturm-Liouville operators. Eur. J. Pure Appl. Math. 3, 831-838 (2010)

[27] Menken, H.: Accurate asymptotic formulas for eigenvalues and eigenfunctions of a boundary-value problem of fourth order. Bound. Value Probl. Article ID: 720235 (2010)

[28] Menken, H., Mamedov, Kh.R.: Basis property in $L_{p}(0,1)$ of the root functions corresponding to a boundary-value problem. J. Appl. Funct. Anal. 5, 351356 (2010)

[29] Mikhailov, V.P.: On Riesz bases in $L_{2}(0,1)$. Dokl. Akad. Nauk SSSR (in Russian) 144(5), 981-984 (1962)

[30] Naimark, M.A.: Linear Differential Operators, 2nd edn. Nauka, Moskow (1969) (in Russian) [English Trans.: Part I, Ungar, New York (1967)]

[31] Shkalikov, A.A.: On the basis property of eigenfunctions of an ordinary differential operator with integral boundary conditions. Vestnik Moskov. Univ. Ser. I Mat. Mekh. (Moscow Univ. Math. Bull.) 6, 12-21 (1982)

[32] Shkalikov, A.A., Veliev, O.A.: On the Riesz basis property of eigen and associated functions of periodic and anti-periodic Sturm-Liouville problems. Math. Notes 85, 647-660 (2009)

[33] Veliev, O.A.: Asymptotic analysis of non-self-adjoint Hill operators. Central Eur. J. Math. 11(12), 2234-2256 (2013)

[34] Veliev, O.A.: On the nonself-adjoint ordinary differential operators with periodic boundary conditions. Isr. J. Math. 176, 195-207 (2010)

[35] Veliev, O.A., Toppamuk Duman, M.: The spectral expansion for a nonselfadjoint Hill operator with a locally integrable potential. J. Math. Anal. Appl. 265, 76-90 (2002)

[36] Zygmund, A.: Trigonometric Series, vol. 2. Cambridge University Press, New York (1959) 
Hikmet Gunes and Nazim B. Kerimov

Department of Mathematics

Mersin University

Mersin 33343, Turkey

e-mail: nazimkerimov@yahoo.com;

hikmetgunes@hotmail.de

Ufuk Kaya

Department of Mathematics

Bitlis Eren University

Bitlis 13000, Turkey

e-mail: mat-ufuk@hotmail.com

Received: December 24, 2014.

Accepted: March 21, 2015. 